\title{
Assembly of an Antiparallel Homo-Adenine DNA Duplex by Small
}

\section{Molecule Binding}

\author{
Özgül Persil, Catherine T. Santai, Swapan S. Jain and Nicholas V. Hud \\ School of Chemistry and Biochemistry, Parker H. Petit Institute of Bioengineering and \\ Biosciences, Georgia Institute of Technology, Atlanta, GA 30332-0400, USA \\ E-mail: hud@chemistry.gatech.edu
}

\section{Supporting Information}

\section{Materials and Methods:}

Sample Preparation. $(\mathrm{dA})_{4}$ and 3'-d(A) $)_{8}-5$ ' -5 ' $-\mathrm{d}(\mathrm{A})_{8}-3$ ' oligodeoxynucleotides were synthesized in house on an automated synthesizer using standard phosphoramidite chemistry. $(\mathrm{dA})_{8},(\mathrm{dA})_{16},(\mathrm{dA})_{32}, \mathrm{~d}\left(\mathrm{GACCCGCA} \mathrm{CCTCGCC}_{8}\right.$ and d(GGCGAGGA ${ }_{8}$ GCGGGTC) oligonucleotides were purchased from IDT (Coralville, IA). Full-length oligonucleotides were separated from failure sequences by denaturing polyacrylamide gel electrophoresis. Full-length products were extracted from the gel matrix using the crush-and-soak method followed by ethanol precipitation and desalting by passage over a $1 \mathrm{~m} \mathrm{G}-25$ sephadex column. Column fractions containing purified oligonucleotides were pooled, lyophilized and resuspended in $\mathrm{dH}_{2} \mathrm{O}$. Coralyne chloride and proflavine hemisulfate were purchased from Sigma and used without further purification.

Oligonucleotide and small molecule concentrations were determined by UV-Vis spectroscopy using the following extinction coefficients: $(\mathrm{dA})_{4}, \varepsilon_{260}=51400 \mathrm{M}^{-1} \mathrm{~cm}^{-1} ;(\mathrm{dA})_{8}$, $\varepsilon_{260}=99400 \mathrm{M}^{-1} \mathrm{~cm}^{-1} ;(\mathrm{dA})_{16}, \varepsilon_{260}=195400 \mathrm{M}^{-1} \mathrm{~cm}^{-1} ;(\mathrm{dA})_{32}, \varepsilon_{260}=387400 \mathrm{M}^{-1} \mathrm{~cm}^{-1}$;

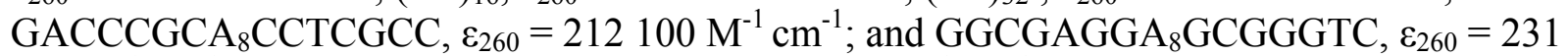
$900 \mathrm{M}^{-1} \mathrm{~cm}^{-1} ; 3^{\prime}-\mathrm{d}(\mathrm{A})_{8}{ }^{-5}{ }^{\prime}-5^{\prime}-\mathrm{d}(\mathrm{A})_{8^{-}} 3^{\prime}, \varepsilon_{260}=195400 \mathrm{M}^{-1} \mathrm{~cm}^{-1}$; coralyne chloride, $\varepsilon_{420}=14500$ $\mathrm{M}^{-1} \mathrm{~cm}^{-1}$; proflavine hemisulfate, $\varepsilon_{444}=38900 \mathrm{M}^{-1} \mathrm{~cm}^{-1}$.

Polyacrylamide gel electrophoresis (PAGE). Oligonucleotides were 5'-end labeled using $\gamma-{ }^{32} \mathrm{P}-\mathrm{ATP}$ (ICN) and T4 polynucleotide kinase (New England Biolabs). Non-denaturing PAGE experiments were run in a standard $1 \times$ TBE buffer (Tris-Borate-EDTA), at a constant power of $7 \mathrm{~W}$ and an ambient temperature of $4^{\circ} \mathrm{C}$. Gels were imaged using a Fuji Phosphor Imager (FLA-3000).

Circular dichroism (CD) and UV-Vis spectrophotometry. CD spectra were acquired on a JASCO J-810 CD spectropolarimeter equipped with Peltier temperature control unit. Spectra were acquired using a $1 \mathrm{~cm}$ path length cell. UV-Vis absorbance measurements were performed using a HP $8453 \mathrm{UV}$-Vis diode array spectrophotometer equipped with an Agilent 89090A Peltier temperature control unit. UV melting profiles were acquired by increasing the sample temperature at a rate of $1^{\circ} \mathrm{C} \mathrm{min}^{-1}$ from 5 to $80^{\circ} \mathrm{C}$. 
Atomic Force Microscopy (AFM). Scanning force images were acquired using a Nanoscope IIIa AFM (Digital Instruments) equipped with a J scanner operating in tapping mode. AFM tips were NSC12 non-contact silicon rectangular cantilevers (Mikromasch USA, Portland), which were cleaned with ozone prior to use. Samples $(20 \mu \mathrm{l})$ were deposited onto freshly cleaved mica, incubated for 30 min at $4{ }^{\circ} \mathrm{C}$, rinsed once with $\mathrm{dH}_{2} \mathrm{O}\left(4^{\circ} \mathrm{C}\right)$, wicked dry by touching an edge of the mica to filter paper, blown dry with nitrogen gas and stored overnight in a vacuum desiccator at room temperature. Samples were imaged under ambient conditions. Images were flattened to remove background slope in the horizontal dimensions. 

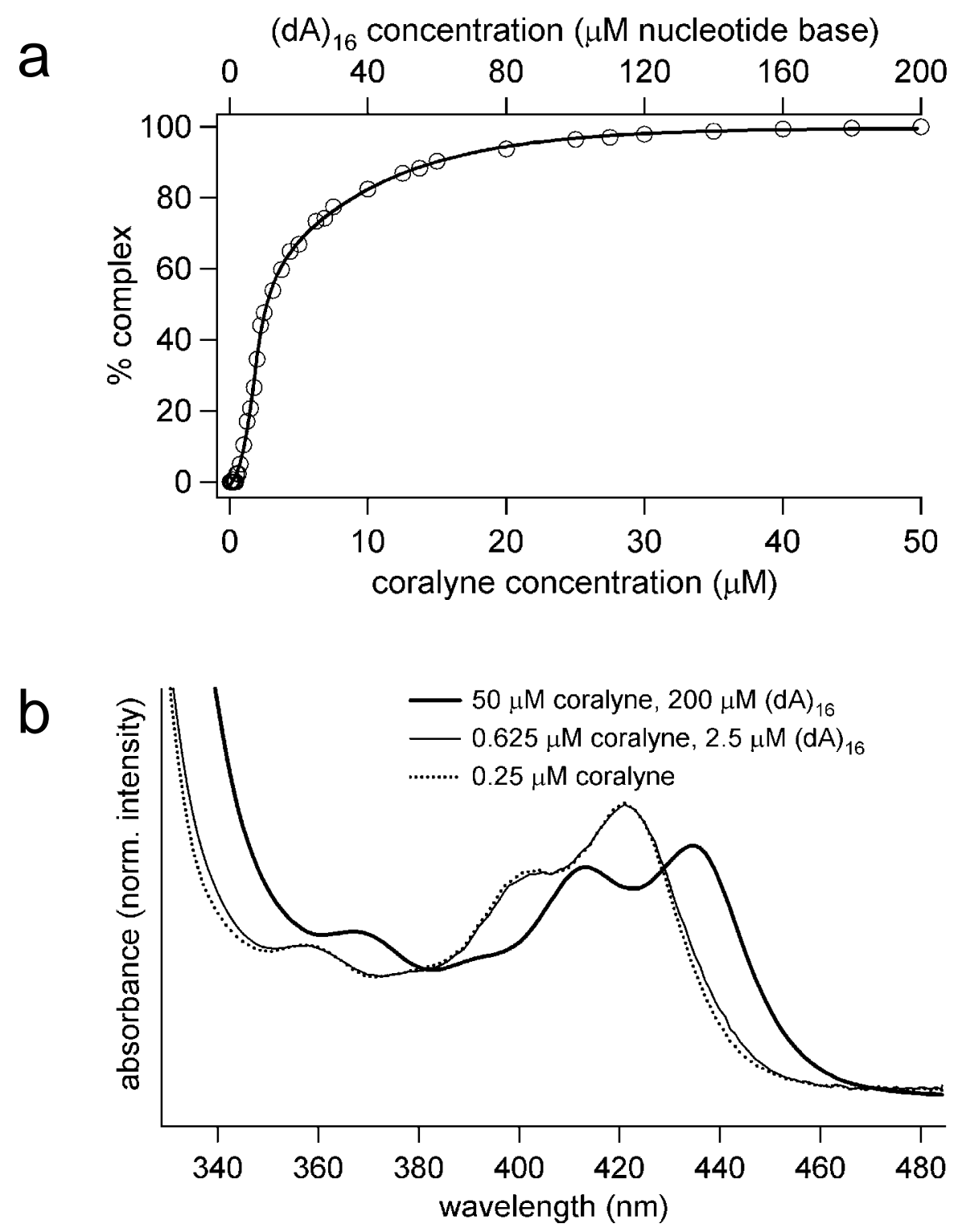

Figure S1. (a) Plot of percent (dA) 16 -coralyne complex formed as a function of DNA and coralyne concentration. The relative concentration of coralyne to (dA) $)_{16}$ was one coralyne molecule per four nucleotide base for all data points, the stoichiometry previously determined for the poly(dA)-coralyne complex (Polak and Hud, Nucleic Acids Res. 2002, 30, 983-992). Percent of $(\mathrm{dA})_{16}$-coralyne complex formed at each concentration was determined by performing a leastsquares fit of the corresponding UV absorption spectrum as a weighted sum of two absorption spectra, which were the spectrum of $200 \mu \mathrm{M}(\mathrm{dA})_{16}$ (in nucleotide base), $50 \mu \mathrm{M}$ coralyne and a spectrum of $0.025 \mu \mathrm{M}$ coralyne (i.e. free coralyne with no DNA). All samples contained 115 $\mathrm{mM} \mathrm{NaCl}$ and $13 \mathrm{mM} \mathrm{NaCacodylic,} \mathrm{pH}$ 6.8. Spectra were acquired at $22^{\circ} \mathrm{C}$. (b) $\mathrm{UV}$ absorbance spectra representative of those used to generate the plot in $\mathbf{a}$. The spectral region from 340 to 375 $\mathrm{nm}$ was that used to determine the fraction of $(\mathrm{dA})_{16}$-coralyne complex formed. 


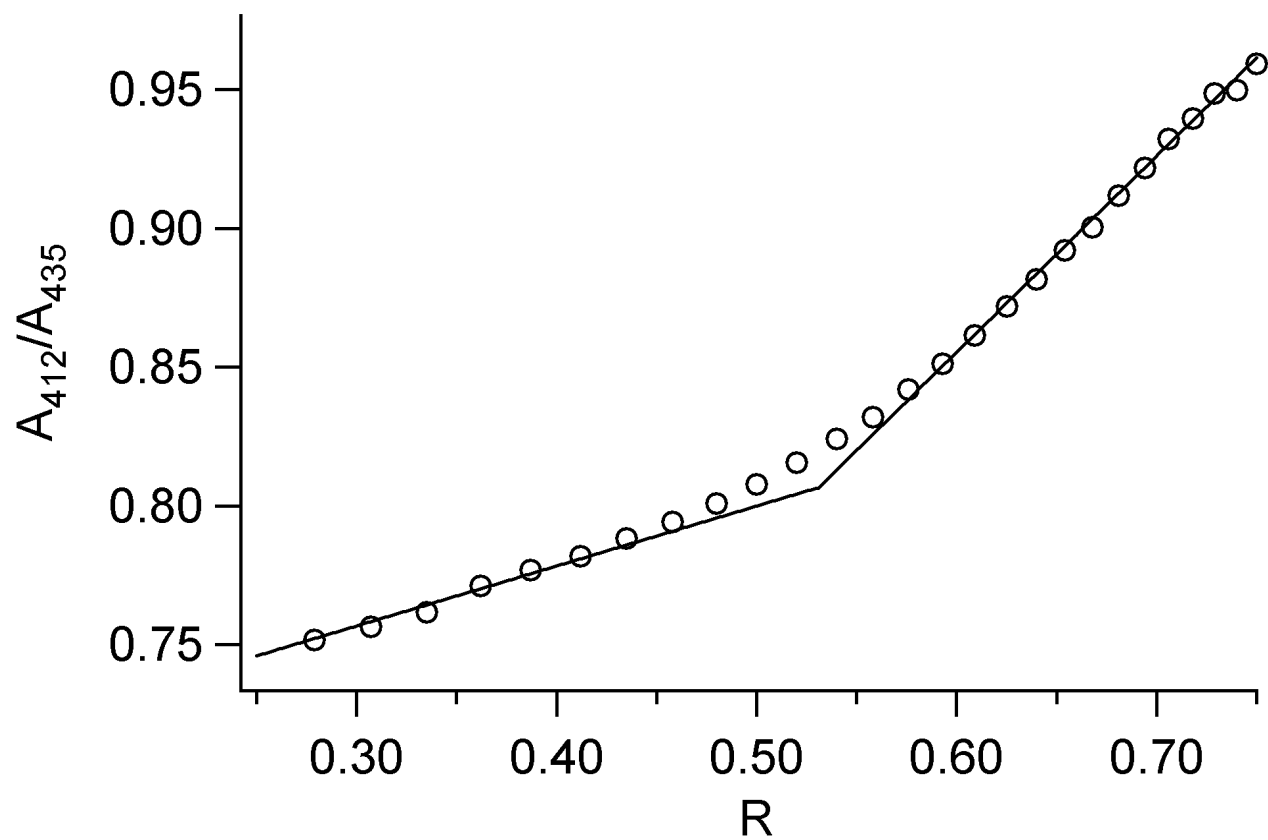

Figure S2. Job Plot analysis of plusA8·minusA8 with coralyne. A412/A435 is the ratio of coralyne absorbance at $412 \mathrm{~nm}$ versus $435 \mathrm{~nm} . \mathrm{R}=$ [coralyne]/([coralyne] $+[(\mathrm{dA}) / 4])$. The combined concentration of coralyne and $(\mathrm{dA}) / 4$ was $15 \mu \mathrm{M}$ for each data point in the Job plot.

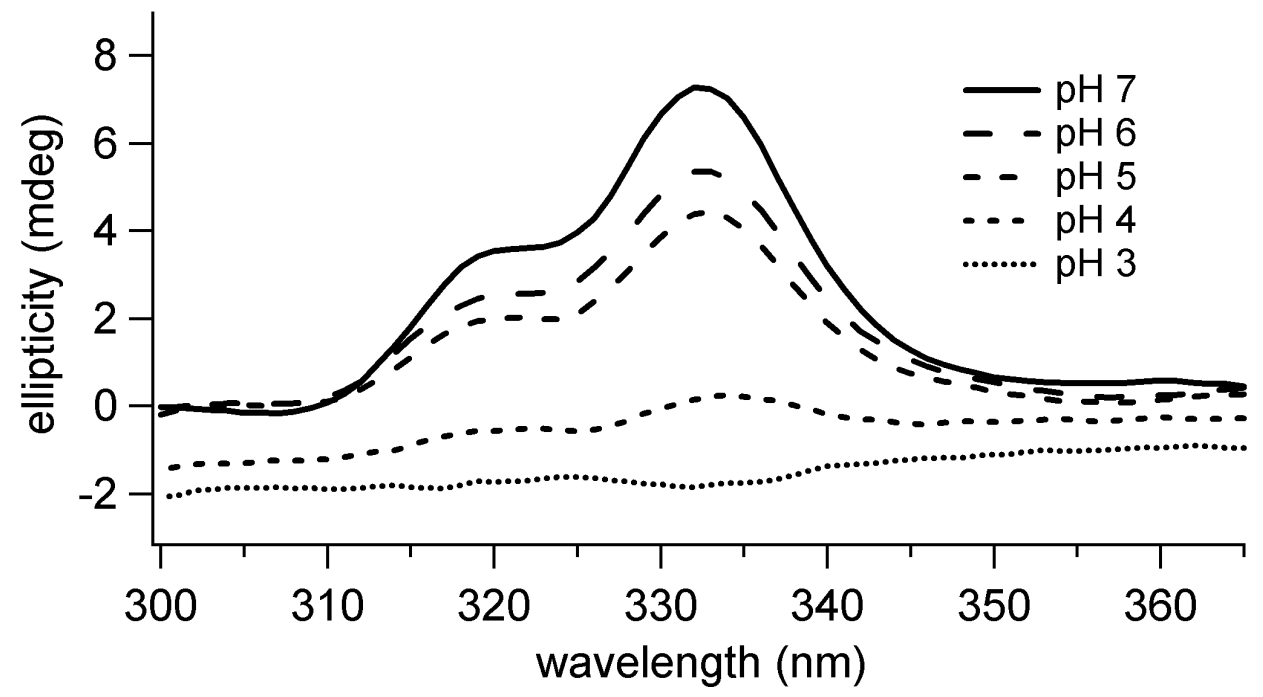

Figure S3. The 300 to $365 \mathrm{~nm}$ region of $\mathrm{CD}$ spectra of $(\mathrm{dA})_{32}$ with coralyne at various $\mathrm{pH}$ values. The reduction of the positive $\mathrm{CD}$ bands in this region at lower $\mathrm{pH}$ indicates the reduced stability of the $(\mathrm{dA})_{32}$-coralyne complex with decreasing $\mathrm{pH}$. Samples were $55 \mu \mathrm{M}$ nucleotide base, $14 \mu \mathrm{M}$ coralyne, $115 \mathrm{mM} \mathrm{NaCl}$ and $13 \mathrm{mM} \mathrm{NaCacodylic.} \mathrm{Samples} \mathrm{with} \mathrm{pH}$ lower than 7 were prepared by the titration of a $\mathrm{pH} 7$ sample with $1 \mathrm{M} \mathrm{HCl}$. 